\title{
Interunit Handoffs From Emergency Department to Inpatient Care: A Cross-Sectional Survey of Physicians at a University Medical Center
}

\author{
Christopher J. Smith, MD*, Denise H. Britigan, PhD, MA, CHES², Elizabeth Lyden, MS ${ }^{3}$, Nathan Anderson, MD4, \\ Ted J. Welniak, MD5, Michael C. Wadman, MD ${ }^{6}$
}

\begin{abstract}
${ }^{1}$ Department of Internal Medicine, Division of General Internal Medicine, University of Nebraska Medical Center College of Medicine, Omaha, Nebraska; '2Department of Health Promotion, Social, and Behavioral Health, University of Nebraska Medical Center College of Public Health, Omaha, Nebraska; ${ }^{3}$ Department of Biostatistics, University of Nebraska Medical Center College of Public Health, Omaha, Nebraska; ${ }^{4}$ Department of Internal Medicine, University of Nebraska Medical Center, Omaha, Nebraska; ${ }^{5}$ Department of Emergency Medicine, Maimonides Medical Center, Brooklyn, New York; ' University of Nebraska Medical Center College of Medicine and Department of Emergency Medicine, University of Nebraska Medical Center, Omaha, Nebraska.
\end{abstract}

BACKGROUND: Emergency department (ED) to inpatient physician handoffs are subject to complex challenges. We assessed physicians' perceptions of the ED admission handoff process and identified potential barriers to safe patient care.

METHODS: We conducted a cross-sectional survey at a 627-bed tertiary care academic medical center. Eligible participants included all resident, fellow, and faculty physicians directly involved in admission handoffs from emergency medicine (EM) and 5 medical admitting services. The survey addressed communication quality, clinical information, interpersonal perceptions, assignment of responsibilities, organizational factors, and patient safety. Participants reported their responses via a 5-point Likert scale and an open-ended description of handoff-related adverse events.

RESULTS: Response rates were $63 \%$ for admitting (94/150) and $86 \%$ for EM physicians (32/37). Compared to EM respondents, admitting physicians reported that vital clinical information was communicated less frequently for all 8 content areas $(P<0.001)$. Ninety-four percent of EM physicians felt defensive at least "sometimes." Twenty-nine percent of all respondents reported handoff-related adverse events, most frequently related to ineffective communication. Sequential handoffs were common for both EM and admitting services, with $78 \%$ of physicians reporting they negatively impacted patient care.

CONCLUSION: Physicians reported that patient safety was often at risk during the ED admission handoff process. Admitting and EM physicians had divergent perceptions regarding handoff communication, and sequential handoffs were common. Further research is needed to better understand this complex process and to investigate strategies for improvement. Journal of Hospital Medicine 2015; 10:711-717. (C) 2015 Society of Hospital Medicine
Handoffs are the exchange of information between health professionals that accompany the transfer of patient-care responsibility. ${ }^{1}$ Poor handoff practices are associated with unsafe and inefficient care. ${ }^{2-4}$ Teaching hospitals are especially at risk, as resident work-hour restrictions have increased the number of handoffs. ${ }^{5}$ Accreditation agencies now require that hospitals and residency programs have structured handoff processes ${ }^{6,7}$ and that medical students ${ }^{8}$ and residents $^{9-12}$ demonstrate competency in handoffs.

Physician handoff research has primarily focused on handoffs within a service or discipline. These "withinunit" handoffs should be differentiated from "interunit" handoffs. ${ }^{13,14}$ Interunit handoffs, such as the transition from the emergency department (ED) to inpatient setting, are subject to unique challenges. The ED admission

\footnotetext{
*Address for correspondence and reprint requests: Christopher J. Smith, MD, 986430 Nebraska Medical Center, Omaha, NE 68198-6430; Telephone: 402-559-7496; Fax: 402-559-5588; E-mail: csmithj@unmc.edu

Additional Supporting Information may be found in the online version of this article.

Received: April 9, 2015; Revised: June 24, 2015; Accepted: June 27, 2015

2015 Society of Hospital Medicine DOI 10.1002/jhm.2431

Published online in Wiley Online Library (Wileyonlinelibrary.com).
}

process involves changes in personnel, provider specialty, and location. ${ }^{15}$ The transition occurs when the patient's clinical trajectory is uncertain, treatments are being initiated, and test results are pending. Other barriers include interdisciplinary cultural differences, interphysician conflict, unstructured communication, environmental factors, and complex care coordination. ${ }^{13-18}$ Despite these challenges, there is relatively little research specifically examining ED to inpatient handoffs, and most of what is available has focused on individual services within an institution. $^{13-15,18-25}$

As part of an institutional effort to improve our ED admission handoff practices, we conducted a crosssectional, survey-based needs-assessment involving emergency medicine (EM) and 5 inpatient medical services. The objective of this study was to determine physicians' perceptions of the ED admission handoff process and to identify potential barriers to safe patient care.

\section{METHODS}

\section{Survey Design}

A study group comprised of resident and faculty physicians in internal medicine (IM) and EM, as well as a healthcare communication expert, designed analogous cross-sectional surveys to determine the 
perceptions of admitting (see Supporting Information, Appendix 1, in the online version of this article) and EM (see Supporting Information, Appendix 2, in the online version of this article) physicians toward the admission handoff process. Using an iterative process to ensure content validity, we created questions in 6 domains based on the expert opinion of the authors and emergent themes identified in the literature. ${ }^{15,19,22,24}$ These themes were general communication quality, clinical information, interpersonal perceptions, responsibilities, organizational factors, and patient safety. We asked respondents to report their answers using 5-point Likert and Likert-like scales. Questions regarding frequency were assigned semiquantitative values: rarely $=$ $0 \%$ to $24 \%$, sometimes $=25 \%$ to $49 \%$, often $=50 \%$ to $74 \%$, very often $75 \%$ to $99 \%$, and always $=100 \%$. We also asked an open-ended question, asking respondents to describe any handoff-related adverse events (defined as patient harm or near miss) they encountered in the past 3 months. We pilot tested the survey for clarity and relevance prior to distribution on a group of 5 physicians from the participating services. The institutional internal review board approved the protocol (\#046-13-EX).

\section{Setting, Participants, and Recruitment}

We conducted the study at a 627-bed tertiary care academic medical center. Eligible participants included all resident, fellow, and faculty physicians directly involved in admission handoffs from EM and 5 medical inpatient services (university-based IM, universitybased family medicine [FM], community-based FM, cardiology, and critical care medicine). The admitting services accounted for two-thirds of the institution's 10,000 annual adult, nonobstetric ED admissions. Physicians who had not participated in admission handoffs in the past 3 months were excluded.

At the time of the study, there was no standardized institutional process for admission handoff communication, nor was there policy delineating when patient-care responsibility transferred from the EM to admitting physician. The admission handoff process generally relied on verbal handoff via telephone between EM and admitting physicians. All services used the same electronic health record, but there was no written handoff note, and EM physician documentation generally was not available at the time of handoff. To determine patient assignment schemes following admission handoff, we questioned leadership from the participating admitting services.

We distributed and collected anonymous hard-copy surveys at educational conferences in March 2013. We emailed a link to an online survey to eligible participants who could not be reached in person. Subjects voluntarily participated and provided consent via cover letter.

\section{Data Analysis}

We compiled survey data and performed descriptive analysis. We assessed the internal consistency of the survey domains that were made up of at least 3 questions using Cronbach's $\alpha$. To compare the distribution of aggregate admitting service responses to EM responses, we used the Mann-Whitney test. We used the Fisher exact test to examine the associations of dichotomized responses ( $<50 \%$ vs $\geq 50 \%$ ) to the level of training (intern vs resident vs fellow/faculty) and to the admitting service affiliation (university-based IM vs university-based FM vs aggregate of other services). When indicated, we made pairwise comparisons using the Bonferroni method to compute adjusted $P$ values. We analyzed data independently using both SPSS version 20 (IBM Corp., Armonk, NY) and SAS version 9.3 (SAS Inc., Cary, NC) software and considered a $P$ value $<0.05$ to be significant. Three researchers independently categorized descriptions of adverse events based on a previously published qualitative analysis, ${ }^{15}$ with disagreements settled by consensus.

\section{RESULTS}

After applying exclusion criteria, the survey response rate was $63 \%$ for admitting physicians (94/150) and $86 \%$ for EM physicians (32/37). Participants' service affiliation and level of training are shown in the Table 1. Table 2 provides the distribution of survey responses for EM and admitting physicians.

The processes for assigning responsibilities following the initial handoff differed between admitting services, and within a service the process was often dynamic. For example, within the university-based IM and community-based FM services, the assignment process varied depending on timing (day vs night, weekday vs weekend). For the critical care medicine and cardiology services, fellows accepted admission handoff calls, and depending on competing clinical responsibilities and the patient's stability, either evaluated the patient independently or sent a resident to perform a preliminary evaluation. We reviewed and classified these varied admission assignment strategies into 4 general schemes (Figure 1). All 5 admitting services relied partly or entirely on housestaff for receiving admission handoffs, as did the EM service.

\section{Communication Quality and Content}

Cronbach's $\alpha$ was 0.72 for general handoff questions and 0.89 for clinical information questions. Compared with EM respondents, admitting physicians reported worse quality of communication $(P<0.001)$ and less confidence in the handoff system's ability to ensure patient safety $(P=0.04)$. Admitting physicians reported communication of clinical information occurred less frequently than EM physicians for all 8 content areas $(P<$ 0.001 for all). There were no significant differences in responses between various levels of training and service affiliations. 
TABLE 1. Service Affiliation and Level of Training of Survey Respondents

\begin{tabular}{|c|c|c|c|c|c|c|c|c|c|c|c|}
\hline \multirow[b]{3}{*}{ Service Affiliation } & \multicolumn{10}{|c|}{ Level of Training } & \multirow{3}{*}{$\frac{\text { Total }}{\text { No. }}$} \\
\hline & \multicolumn{2}{|c|}{ PGY1 } & \multicolumn{2}{|c|}{ PGY2 } & \multicolumn{2}{|c|}{ PGY3 } & \multicolumn{2}{|c|}{ Fellow } & \multicolumn{2}{|c|}{ Staff } & \\
\hline & No. & $\%$ & No. & $\%$ & No. & $\%$ & No. & $\%$ & No. & $\%$ & \\
\hline \multicolumn{12}{|l|}{ Admitting services } \\
\hline University-based IM & 12 & 32.4 & 7 & 18.9 & 5 & 13.5 & 1 & 2.7 & 12 & 32.4 & 37 \\
\hline University-based FM & 15 & 44.1 & 13 & 38.2 & 5 & 14.7 & 1 & 2.9 & 0 & 0 & 34 \\
\hline Community-based FM & 5 & 50.0 & 1 & 10.0 & 3 & 30.0 & 0 & 0 & 1 & 10.0 & 10 \\
\hline Critical care medicine & 0 & 0 & 0 & 0 & 0 & 0 & 6 & 100.0 & 0 & 0 & 6 \\
\hline Cardiology & 0 & 0 & 0 & 0 & 0 & 0 & 7 & 100.0 & 0 & 0 & 7 \\
\hline Admitting services total & 32 & 34.0 & 21 & 22.3 & 13 & 13.8 & 15 & 16.0 & 13 & 13.8 & 94 \\
\hline Emergency medicine & 6 & 18.8 & 8 & 25.0 & 5 & 15.6 & 0 & 0 & 13 & 40.6 & 32 \\
\hline
\end{tabular}

NOTE: Abbreviations: FM, family medicine; IM, internal medicine; PGY, postgraduate year.

\section{Interpersonal Perceptions}

EM respondents reported admitting physicians asked clinical questions less frequently than did admitting respondents $(P<0.001)$. Ninety-four percent of EM physicians $(\mathrm{n}=30)$ felt they had to defend their clinical decisions at least "sometimes." EM interns $(P=0.009)$ and faculty $(P=0.01)$ were more likely than residents to report feeling defensive. Most admitting physicians $(60 \%, \mathrm{n}=56)$ "often" agreed with decisions made by the EM provider, but $29 \%(n=27)$ agreed less than half the time. One-third of admitting $(\mathrm{n}=31)$ and $16 \%$ of EM physicians $(\mathrm{n}=5)$ reported routine (ie, $>50 \%$ of admissions) meaningful face-toface communication with one another at the time of admission.

\section{Responsibilities}

When asked who was primarily responsible for patients "boarding" in the ED, defined as nonemergent patient care that occurs "after handoff, but before a patient is physically transferred from the ED, $" 37.6 \% \quad(n=47)$ of respondents answered the admitting physician, $21.6 \%(\mathrm{n}=27)$ answered the EM physician, $34.4 \%(\mathrm{n}=43)$ answered both, and $6.4 \%(\mathrm{n}=8)$ answered "don't know." Responses were similar for EM and admitting physicians.

\section{Organizational Factors}

Fifty-six percent of all respondents $(n=69)$ reported they were distracted during handoffs by competing clinical duties $\geq 50 \%$ of the time. Environmental factors, such as noise, more commonly distracted EM physicians $(P=0.001)$. Approximately $60 \%(\mathrm{n}=56)$ of admitting physicians reported using a triage system to distribute admissions, with a resultant $57 \%$ $(\mathrm{n}=32)$ reporting sequential handoffs (ie, handoffs of handoffs) occurred at least "sometimes." About 80\% of EM physicians $(n=23)$ reported that shift change led to sequential handoffs at least "sometimes." Seventy-eight percent $(n=67)$ of physicians felt sequential handoffs had a negative impact on patient care.

\section{Patient Safety}

Thirty-four percent of admitting $(n=30)$ and $19 \%$ of EM physicians $(n=6)$ reported a patient was harmed or suffered a near miss in the past 3 months because of an ineffective handoff, with $58 \%(\mathrm{n}=21)$ reporting $\geq 2$ examples. Twenty-four respondents described 29 adverse events. Respondents described perceived mistakes in diagnosis $(n=11)$, treatment $(n=16)$, and disposition $(n=12)$, with some examples falling into more than 1 category. Absent or ineffective communication contributed to 27 of 29 examples. Other commonly cited areas of vulnerability included uncertain assignment of responsibility, sequential handoffs, and patient boarding.

\section{DISCUSSION}

Based upon physician self-reporting, we identified perceived barriers to safe ED admission handoff across several domains. This study adds to the literature, as it provides a cross-section of multiple inpatient services with varying admission schemes to underscore the complexities facing hospitals in safely transitioning patients between units. As noted in previous studies, one-third of physicians reported a handoff-related adverse event, ${ }^{15}$ and there was significant disagreement between handoff participants about communication of critical information. ${ }^{21,26}$ These differences in perceptions suggest a failure of physicians to accurately transfer information to create a shared understanding of patient care, ${ }^{21}$ which is the central function of handoffs.

EM physicians frequently felt that admitting physicians did not trust their clinical decisions, a perception supported by the fact that over $25 \%$ of admitting respondents' usually disagreed with decisions in the ED. Interdisciplinary trust is central in negotiating a shared plan of care ${ }^{13}$ and mitigating conflict to ensure a safe transition of patient care. ${ }^{16}$ Handoffs are complex social interactions, and feelings of defensiveness and mistrust are likely exacerbated by in-group/outgroup biases, ${ }^{15}$ conflicting information expectations, ${ }^{19}$ 
TABLE 2. Selected Questions and Distribution of Responses from ED Admission Handoff Survey ${ }^{\star}$

\begin{tabular}{|c|c|c|c|c|c|c|c|c|c|c|c|}
\hline \multirow[b]{2}{*}{ Question } & \multirow[b]{2}{*}{ Service } & \multicolumn{2}{|c|}{ Very Poor } & \multicolumn{2}{|c|}{ Poor } & \multicolumn{2}{|c|}{ Fair } & \multicolumn{2}{|c|}{ Good } & \multicolumn{2}{|c|}{ Very Good } \\
\hline & & No. & $\%$ & No. & $\%$ & No. & $\%$ & No. & $\%$ & No. & $\%$ \\
\hline \multirow[t]{2}{*}{ Generally, the quality of communication between EM and admitting physicians is: ${ }^{\dagger}$} & Admitting & 0 & 0 & 8 & $8.6 \%$ & 37 & $39.7 \%$ & 46 & $49.4 \%$ & 2 & $2.1 \%$ \\
\hline & EM & 0 & 0 & 2 & $6.2 \%$ & 4 & $12.5 \%$ & 20 & $62.5 \%$ & 6 & $18.7 \%$ \\
\hline \multirow[t]{2}{*}{ The current handoff system's ability to ensure patient safety is generally: ${ }^{\dagger}$} & Admitting & 1 & $1.0 \%$ & 10 & $10.7 \%$ & 43 & $46.2 \%$ & 37 & $39.7 \%$ & 2 & $2.1 \%$ \\
\hline & EM & 1 & $3.1 \%$ & 1 & $3.1 \%$ & 11 & $34.3 \%$ & 15 & $46.8 \%$ & 4 & $12.5 \%$ \\
\hline \multirow[t]{3}{*}{ The current handoff system's ability to ensure efficient patient care is generally: } & Admitting & 3 & $3.2 \%$ & 20 & $21.7 \%$ & 31 & $33.6 \%$ & 36 & $39.1 \%$ & 2 & $2.1 \%$ \\
\hline & EM & 2 & $6.2 \%$ & 5 & $15.6 \%$ & 15 & $46.8 \%$ & 10 & $31.2 \%$ & 0 & \\
\hline & & & \multicolumn{2}{|c|}{$\begin{array}{c}\text { Rarely } \\
(0 \%-24 \%)\end{array}$} & \multicolumn{2}{|c|}{$\begin{array}{l}\text { Sometimes } \\
(25 \%-49 \%)\end{array}$} & \multicolumn{2}{|c|}{$\begin{array}{c}\text { Often } \\
(50 \%-74 \%)\end{array}$} & $\begin{array}{c}\text { Very Often } \\
(75 \%-99 \%)\end{array}$ & \multicolumn{2}{|c|}{$\begin{array}{l}\text { Always } \\
(100 \%)\end{array}$} \\
\hline
\end{tabular}

During handoff, how often does the EM physician provide the following information to the admitting service? The working diagnosis of the EM physician ${ }^{\dagger}$

Relevant past medical/surgical history
Relevant physical exam findings (including abnormal vital signs) $)^{\dagger}$
Results of relevant diagnostic studies (labs, imaging)
Procedures and therapeutic interventions initiated while in the ED
Trend in the patient's clinical condition while in the ED
Current clinical condition of the patient (at time of handoff)
Pending diagnostic studies (labs, imaging), if ordered

During handoff, how often are clinical questions asked about the patient being admitted?

In general, how often do you agree with the clinical decisions made by the EM physician? ${ }^{\ddagger}$ Generally, how often do you feel you have to defend your clinical decisions to the admitting service? How often do you have clinically meaningful face-to-face communication with the EM/admitting physician about the patient being admitted? On average, how often do competing clinical responsibilities distract you during handoff? On average, how often do environmental factors distract you during handoff?

\begin{tabular}{lcccccccccc} 
Admitting & 5 & $5.4 \%$ & 19 & $20.6 \%$ & 30 & $32.6 \%$ & 30 & $32.6 \%$ & 8 & $8.6 \%$ \\
EM & 0 & & 4 & $12.5 \%$ & 0 & & 12 & $37.5 \%$ & 16 & $50.0 \%$ \\
Admitting & 5 & $5.4 \%$ & 25 & $27.1 \%$ & 40 & $43.4 \%$ & 18 & $19.5 \%$ & 4 & $4.3 \%$ \\
EM & 1 & $3.1 \%$ & 2 & $6.2 \%$ & 5 & $15.6 \%$ & 17 & $53.1 \%$ & 7 & $21.8 \%$ \\
Admitting & 3 & $3.2 \%$ & 25 & $27.1 \%$ & 41 & $44.5 \%$ & 21 & $22.8 \%$ & 2 & $2.1 \%$ \\
EM & 0 & & 5 & $15.6 \%$ & 2 & $6.2 \%$ & 15 & $46.8 \%$ & 10 & $31.2 \%$ \\
Admitting & 2 & $2.1 \%$ & 10 & $10.8 \%$ & 39 & $42.3 \%$ & 37 & $40.2 \%$ & 4 & $4.3 \%$ \\
EM & 1 & $3.1 \%$ & 0 & & 3 & $9.3 \%$ & 14 & $43.7 \%$ & 14 & $43.7 \%$ \\
Admitting & 3 & $3.2 \%$ & 20 & $21.7 \%$ & 34 & $36.9 \%$ & 29 & $31.5 \%$ & 6 & $6.5 \%$ \\
EM & 1 & $3.1 \%$ & 0 & & 3 & $9.3 \%$ & 18 & $56.2 \%$ & 10 & $31.2 \%$ \\
Admiting & 12 & $13.1 \%$ & 27 & $29.6 \%$ & 33 & $36.2 \%$ & 17 & $18.6 \%$ & 2 & $2.1 \%$ \\
EM & 4 & $12.5 \%$ & 1 & $3.1 \%$ & 5 & $15.6 \%$ & 13 & $40.6 \%$ & 9 & $28.1 \%$ \\
Admitting & 3 & $3.2 \%$ & 24 & $26.0 \%$ & 41 & $44.5 \%$ & 18 & $19.5 \%$ & 6 & $6.5 \%$ \\
EM & 1 & $3.1 \%$ & 1 & $3.1 \%$ & 3 & $9.3 \%$ & 13 & $40.6 \%$ & 14 & $43.7 \%$ \\
Admitting & 12 & $13.0 \%$ & 32 & $34.7 \%$ & 29 & $31.5 \%$ & 17 & $18.4 \%$ & 2 & $2.1 \%$ \\
EM & 0 & & 5 & $15.6 \%$ & 6 & $18.7 \%$ & 14 & $43.7 \%$ & 7 & $21.8 \%$ \\
Admitting & 2 & $2.1 \%$ & 1 & $1.0 \%$ & 13 & $14.1 \%$ & 29 & $31.5 \%$ & 47 & $51.0 \%$ \\
EM & 0 & 0 & 5 & $15.6 \%$ & 8 & $25.0 \%$ & 13 & $40.6 \%$ & 6 & $18.7 \%$ \\
Admitting & 1 & $1.0 \%$ & 26 & $27.9 \%$ & 56 & $60.2 \%$ & 10 & $10.7 \%$ & 0 & 0 \\
EM & 2 & $6.2 \%$ & 15 & $46.8 \%$ & 5 & $15.6 \%$ & 10 & $31.2 \%$ & 0 & 0 \\
Admitting & 24 & $25.8 \%$ & 38 & $40.8 \%$ & 22 & $23.6 \%$ & 8 & $8.6 \%$ & 1 & $1.0 \%$ \\
EM & 14 & $43.7 \%$ & 13 & $40.6 \%$ & 4 & $12.5 \%$ & 1 & $3.1 \%$ & 0 & \\
Admitting & 6 & $6.5 \%$ & 34 & $36.9 \%$ & 29 & $31.5 \%$ & 20 & $21.7 \%$ & 3 & $3.2 \%$ \\
EM & 7 & $21.8 \%$ & 8 & $25.0 \%$ & 9 & $28.1 \%$ & 8 & $25.0 \%$ & 0 & 0 \\
Admitting & 44 & $48.3 \%$ & 31 & $34.0 \%$ & 10 & $10.9 \%$ & 6 & $6.5 \%$ & 0 & 0 \\
EM & 7 & $21.8 \%$ & 11 & $34.3 \%$ & 8 & $25.0 \%$ & 4 & $12.5 \%$ & 2 & $6.2 \%$ \\
& & & & & & & & & & \\
\hline & & & & & & & & & &
\end{tabular}

NOTE: Abbreviations: ED, emergency department, EM, Emergency Medicine. *Some rows contain fewer than 126 responses because of missing item-level data. 'Responses of EM and admitting physicians were significantly different $(P<0.05)$. ${ }^{\ddagger}$ Question asked of either EM or admitting physicians, so responses could not be compared.

and discordant ways of interpreting and framing handoff interactions. ${ }^{13}$ Interestingly, EM residents were less likely than interns or faculty to report feeling defensive. This may be in part because residents from EM and admitting services develop relationships during interdisciplinary rotations, which may help facilitate future handoff interactions. ${ }^{27}$ The fact that EM respondents felt defensive, despite reporting less-frequent questioning than admitting physicians, suggests that tone and content of questions played an important role. These findings support the importance of interdisciplinary education and standardization of handoff communication between ED and admitting physicians. ${ }^{23}$ Beach and colleagues have recommended a conceptual framework for interunit handoffs between EM and hospital physicians, but further research is needed to measure its impact in real-world settings. ${ }^{14}$

We also found great variability in admitting services' processes for assigning patient-care responsibility following the initial handoff. Even within an individual service, these processes were often dynamic and relied on physicians at different levels of training. This has several potential consequences. First, it may be difficult for physicians engaged in a handoff to know the level of experience and expertise of one another. These contextual variables play an important role in how handoff information is conveyed, as less experienced clinicians may require explicit information that a more experienced provider may infer. ${ }^{1,21}$ Second, the variability in admission assignment processes may 


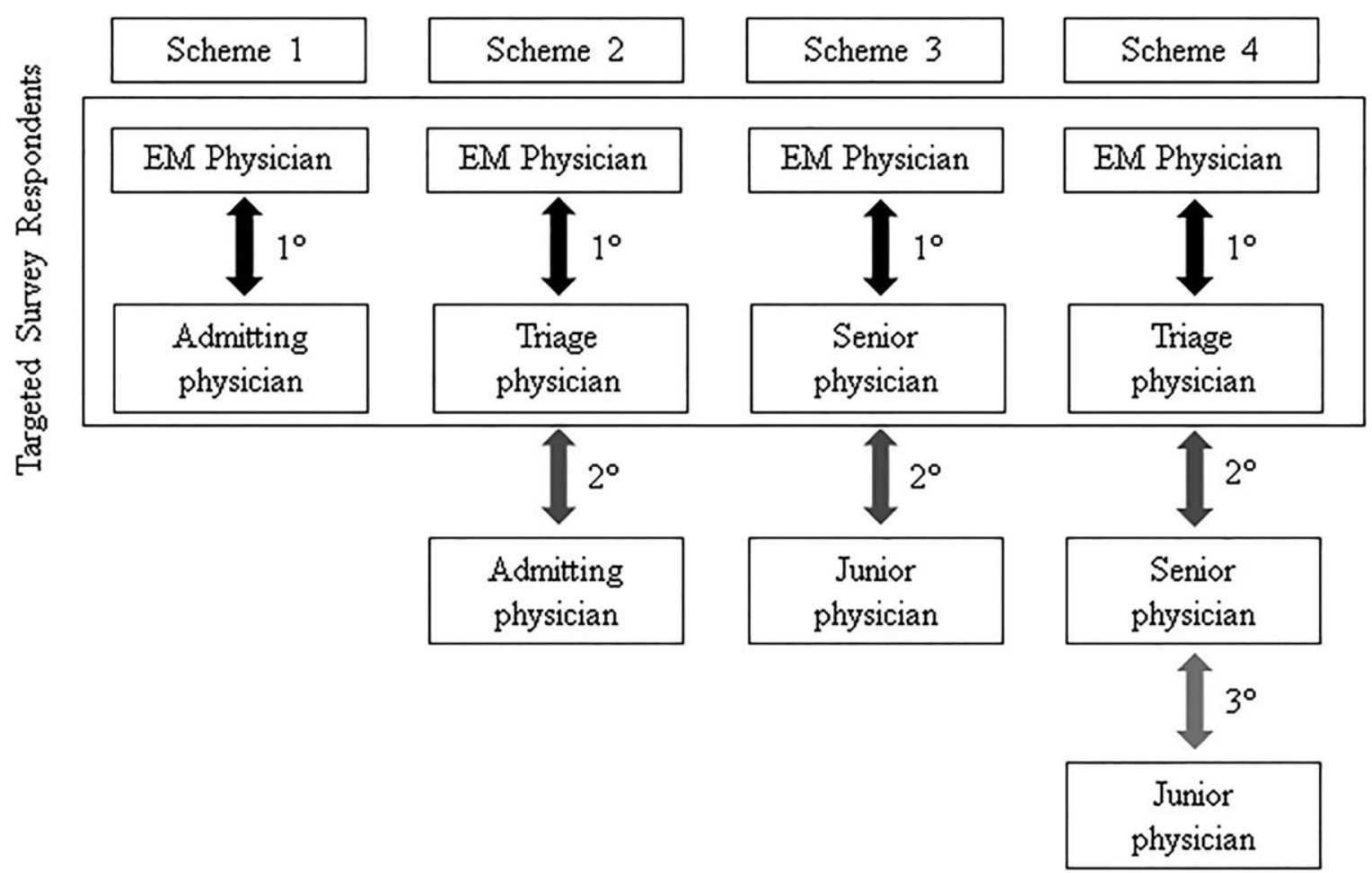

FIG. 1. Emergency department admission assignment schemes for surveyed admitting services. Targeted survey respondents included those physicians who directly participated in admission handoff communication with the emergency medicine (EM) physician (box). Several services employed different processes depending on certain variables (eg, time of day). The numbers next to the double-sided arrows represent the degrees of separation from the initial handoff communication with the emergency medicine physician.

further exacerbate uncertainty regarding responsibility for patients boarding in the ED, making it increasingly difficult for nurses and ancillary staff to know which physician is ultimately responsible for patient care. Finally, the diversity of admission schemes may complicate the development of standardized interunit handoff protocols, policy, and education.

A related finding was that sequential handoffs were common within both EM and admitting services. EM shift handoffs have their own set of barriers, ${ }^{28}$ which can lead to ineffective communication. ${ }^{29}$ Likewise, about two-thirds of admitting respondents reported using an admission triage system. The goal of such systems is to simplify complex call schedules and diverse patient assignment schemes within admitting services, thus streamlining the admission process. These systems may also allow for more consistency in the quality of handoff communication through the creation of triage "specialists." These potential advantages need to be weighed against the increased risk of communication breakdown. The introduction of sequential handoffs creates a game of "telephone," in which there is no direct communication between the first and final caregivers (Figure 1), allowing misinformation to be propagated forward. ${ }^{30}$ Sequential handoffs contributed to several reported adverse events, and the majority of surveyed physicians felt they negatively impacted patient care. Further research is necessary to determine the impact of centralized triage systems and to explore strategies to mitigate informa- tion decay that results from sequential handoffs, as quality-improvement interventions may be of limited benefit if downstream communication remains ineffective. Potential strategies may include standardizing sequential handoff communication, leveraging centralized handoff notes within electronic health records, or developing handoff systems that ensure direct communication between the EM physician and the ultimate admitting provider.

\section{Limitations}

This was a single-institution study, so results may not be generalizable, as handoff processes vary among hospitals. ${ }^{24}$ Our study relied on a novel survey instrument, for which validity and reliability are uncertain, although internal consistency was good for domains that could be tested (Cronbach's $\alpha$ 0.72-0.89). As with other survey-based studies, participant selection, hindsight, recall, and response biases may have influenced the results. We attempted to minimize these risks by pilot testing the survey, targeting a relatively large number of respondents across multiple services, and by making efforts to maximize the response rate by contacting eligible participants both in person and via email. Because results reflect self-reported perceptions, we cannot prove that the factors studied are actually associated with adverse outcomes, nor can we quantify their relative importance. Nevertheless, the reported perceptions raise concerns that warrant further study. 


\section{FUTURE DIRECTIONS}

Further research is needed to examine interventions that may improve clinically relevant outcomes. Development of structured admission handoff protocols should be collaborative ${ }^{31}$ and focus on clinical judgment, rather than rote recitation of data. ${ }^{14}$ Based on our study findings, we are pilot testing a standardized approach for ED-to-hospital handoffs, and portions of this survey will be repeated in the postintervention assessment.

At our institution, housestaff at all levels of training regularly participated in the handoff process. The Accreditation Council for Graduate Medical Education requires that residents demonstrate competence in performing handoffs, ${ }^{7}$ yet handoff training and assessment are inconsistent, ${ }^{23,32,33}$ and published interventions have focused primarily on within-unit handoffs. ${ }^{34-36}$ Additional training should focus on the unique aspects of interunit handoffs. Approaches could include interprofessional communication training, simulation training, and enhanced assessment methods. Additionally, increasing face-to-face communication, perhaps as part of bedside handoffs, could improve relationships and the development of a shared mental model of patient care. More direct involvement by attending physicians will also be important, as there is evidence that such oversight may improve training ${ }^{36}$ and safety, ${ }^{37}$ as more experienced physicians better integrate handoff information. ${ }^{21}$

\section{CONCLUSION}

We identified several perceived barriers to safe interunit handoff from the ED to the inpatient setting. Handoff-related adverse events, a pattern of conflicting physician perceptions, and frequent sequential handoffs were of particular concern. Our findings support the need for collaborative efforts to improve interdisciplinary communication.

Disclosure: Nothing to report.

\section{References}

1. Cohen MD, Hilligoss PB. Handoffs in hospitals: a review of the literature on information exchange while transferring patient responsibility or control. Available at: http://deepblue.lib.umich.edu/handle/2027. 42/61498. Updated 2009. Accessed May 15, 2014.

2. Kitch BT. Handoffs causing patient harm: a survey of medical and surgical house staff. Jt Comm J Qual Patient Saf. 2008;34(10):563-570.

3. Horwitz LI. Consequences of inadequate sign-out for patient care. Arch Intern Med. 2008;168(16):1755-1760.

4. Ong MS, Coiera E. A systematic review of failures in handoff communication during intrahospital transfers. Jt Comm J Qual Patient Saf. 2011;37(6):274-284.

5. Vidyarthi AR, Arora V, Schnipper JL, Wall SD, Wachter RM. Managing discontinuity in academic medical centers: strategies for a safe and effective resident sign-out. J Hosp Med. 2006;1(4):257-266.

6. Arora V, Johnson J. A model for building a standardized hand-off protocol. Jt Comm J Qual Patient Saf. 2006;32(11):646-655.

7. Accreditation Council for Graduate Medical Education. ACGME common program requirements. Available at: https://www.acgme.org/ acgmeweb/Portals/0/PFAssets/ProgramRequirements/CPRs2013.pdf. Updated 2013. Accessed May 7, 2014.

8. Association of American Medical Colleges. Core entrustable professional activities for entering residency. Available at: https://members. aamc.org/eweb/upload/Core\%20EPA\%20Faculty\%20and\%20Learner\% 20Guide.pdf. Updated 2014. Accessed July 7, 2014.
9. Accreditation Council for Graduate Medical Education and American Board of Internal Medicine. The internal medicine milestones. Available at: http://www.acgme.org/acgmeweb/portals/0/pdfs/milestones/ internalmedicinemilestones.pdf. Updated 2012. Accessed December $23,2013$.

10. Accreditation Council for Graduate Medical Education and American Board of Emergency Medicine. The emergency medicine milestones. Available at: https://www.abem.org/public/docs/default-source/migrateddocuments-and-files/em-milestones.pdf?sfvrsn=4. Updated 2012. Accessed December 23, 2013.

11. Accreditation Council for Graduate Medical Education and American Board of Family Medicine. The family medicine milestone project. Available at: http://www.acgme.org/acgmeweb/Portals/0/PDFs/Mile stones/FamilyMedicineMilestones.pdf. Updated 2013. Accessed October 31, 2014.

12. Accreditation Council for Graduate Medical Education and American Board of Pediatrics. The pediatrics milestone project. Available at: http:// acgme.org/acgmeweb/Portals/0/PDFs/Milestones/PediatricsMilestones. pdf. Updated 2013. Accessed October, 31, 2014.

13. Hilligoss B, Cohen MD. The unappreciated challenges of betweenunit handoffs: negotiating and coordinating across boundaries. Ann Emerg Med. 2013;61(2):155-160.

14. Beach C, Cheung DS, Apker J, et al. Improving interunit transitions of care between emergency physicians and hospital medicine physicians: a conceptual approach. Acad Emerg Med. 2012;19(10):1188-1195.

15. Horwitz LI, Meredith T, Schuur JD, Shah NR, Kulkarni RG, Jenq GY. Dropping the baton: a qualitative analysis of failures during the transition from emergency department to inpatient care. Ann Emerg Med. 2009;53(6):701-710.e4.

16. Chan T, Bakewell F, Orlich D, Sherbino J. Conflict prevention, conflict mitigation, and manifestations of conflict during emergency department consultations. Acad Emerg Med. 2014;21(3): 308-313.

17. Kessler CS, Chan T, Loeb JM, Malka ST. I'm clear, you're clear, we're all clear: improving consultation communication skills in undergraduate medical education. Acad Med. 2013;88(6):753-758.

18. Matthews AL, Harvey CM, Schuster RJ, Durso FT. Emergency physician to admitting physician handovers: an exploratory study. Proc Hum Factors Ergon Soc Annu Meet. 2002;46(16): 1511-1515.

19. Apker J, Mallak LA, Gibson SC. Communicating in the "gray zone": perceptions about emergency physician hospitalist handoffs and patient safety. Acad Emerg Med. 2007;14(10):884-894.

20. Hilligoss B, Zheng K. Chart biopsy: an emerging medical practice enabled by electronic health records and its impacts on emergency department-inpatient admission handoffs. J Am Med Inform Assoc. 2013;20(2):260-267.

21. Brannen ML, Cameron KA, Adler M, Goodman D, Holl JL. Admission handoff communications: clinician's shared understanding of patient severity of illness and problems. J Patient Saf. 2009;5(4):237242.

22. Apker J, Mallak LA, Applegate EB III, et al. Exploring emergency physician-hospitalist handoff interactions: development of the handoff communication assessment. Ann Emerg Med. 2010;55(2): $161-170$.

23. Kessler C, Scott NL, Siedsma M, Jordan J, Beach C, Coletti CM. Interunit handoffs of patients and transfers of information: a survey of current practices. Ann Emerg Med. 2014;64(4):343-349.e5.

24. Behara R, Wears RL, Perry SJ, et al. A conceptual framework for studying the safety of transitions in emergency care. In: Henriksen K, Battles JB, Marks ES, Lewin DI, eds. Advances in Patient Safety: From Research to Implementation. Vol. 2: Concepts and Methodology. Rockville, MD: Agency for Healthcare Research and Quality; 2005: 309-321.

25. Gonzalo JD, Yang JJ, Stuckey HL, Fischer CM, Sanchez LD, Herzig SJ. Patient care transitions from the emergency department to the medicine ward: evaluation of a standardized electronic signout tool. Int J Qual Health Care. 2014;26(4):337-347.

26. Chang VY, Arora VM, Lev-Ari S, D'Arcy M, Keysar B. Interns overestimate the effectiveness of their hand-off communication. Pediatrics. 2010;125(3):491-496.

27. Chan T, Sabir K, Sanhan S, Sherbino J. Understanding the impact of residents' interpersonal relationships during emergency department referrals and consultations. J Grad Med Educ. 2013;5(4): 576-581.

28. Cheung DS, Kelly JJ, Beach C, et al. Improving handoffs in the emergency department. Ann Emerg Med. 2010;55(2):171-180.

29. Maughan BC, Lei L, Cydulka RK. ED handoffs: observed practices and communication errors. Am J Emerg Med. 2011;29(5):502511.

30. Jensen AM, Sanders C, Doty J, Higbee D, Rawlings AL. Characterizing information decay in patient handoffs. J Surg Educ. 2014;71(4): 480-485.

31. Pollack CV Jr, Amin A, Talan DA. Emergency medicine and hospital medicine: a call for collaboration. J Emerg Med. 2012;43(2): 328-334. 
32. Kessler C, Shakeel F, Hern HG, et al. A survey of handoff practices in emergency medicine. Am J Med Qual. 2014;29(5):408-414.

33. Horwitz LI. Transfers of patient care between house staff on internal medicine wards: a national survey. Arch Intern Med. 2006;166(11): 1173-1177.

34. Graham KL, Marcantonio ER, Huang GC, Yang J, Davis RB, Smith CC. Effect of a systems intervention on the quality and safety of patient handoffs in an internal medicine residency program. $J$ Gen Intern Med. 2013;28(8):986-993.
35. Starmer AJ, Sectish TC, Simon DW, et al. Rates of medical errors and preventable adverse events among hospitalized children following implementation of a resident handoff bundle. JAMA. 2013;310(21): 2262-2270.

36. Chu ES, Reid M, Schulz T, et al. A structured handoff program for interns. Acad Med. 2009;84(3):347-352.

37. Nabors C, Peterson SJ, Lee WN, et al. Experience with faculty supervision of an electronic resident sign-out system. Am J Med. 2010; 123(4):376-381. 